\title{
Diagnosing scrub typhus without eschar: a case report using metagenomic next-generation sequencing (mNGS)
}

\author{
Jie Li ${ }^{1 \#}$, Changwen Chen ${ }^{2 *}$, Fangqiang Zou ${ }^{2 *}$, Lin Liu ${ }^{2 *}$, Baolong Wang ${ }^{2 *}$, Huan Kang ${ }^{2 *}$, Binsen Liu ${ }^{3}$, \\ Shouhong Wang ${ }^{1}$, Tiehe Qin ${ }^{1}$ \\ ${ }^{1}$ Department of Critical Care Medicine, Guangdong Provincial Geriatrics Institute, Guangdong Provincial People’s Hospital, Guangdong Academy \\ of Medical Sciences, Guangzhou, China; ${ }^{2}$ Department of Critical Care Medicine, Guangdong Provincial People's Hospital Ganzhou Hospital, \\ Ganzhou Municipal Hospital, Guangzhou, China; ${ }^{3}$ Department of Critical Care Medicine, People's Hospital of Shangyou County, Ganzhou, China \\ \#These authors contributed equally to this work and are first co-authors. \\ *These authors are second co-authors. \\ Correspondence to: Shouhong Wang; Tiehe Qin. Department of Critical Care Medicine, Guangdong Provincial Geriatrics Institute, Guangdong \\ Provincial People's Hospital, Guangdong Academy of Medical Sciences, Guangzhou, China. Email: wshdoctor@139.com; qth6626@163.com; Binsen \\ Liu. Department of Critical Care Medicine, People's Hospital of Shangyou County, Ganzhou, China. Email: 403124360@qq.com.
}

\begin{abstract}
Scrub typhus is a disease caused by the bacteria, Orientia tsutsugamushi, which is spread to people through the bites of infected larval mites. Symptoms include eschar at the place of infection, as well as many flu-like symptoms, e.g., fever, headache, chills and skin rash. As eschar is the most typical symptom of scrub typhus, it is often used to diagnose the disease, but if a patient does not display an obvious eschar lesion, diagnosing the disease can prove to be difficult. To help improve the diagnoses of scrub typhus, metagenomic next-generation sequencing (mNGS) has been used as a new approach to identifying pathogens. Here, we report a 51-year-old patient who had unexplained fever for a week and was admitted to hospital with no obvious eschar on her body. Smears and cultures of blood and sputum samples were first performed, but all returned a negative result for scrub typhus. We then conducted a mNGS analysis of blood and sputum samples and were able to identify the pathogenic microbe. Subsequently, a total of 377 reads, as well as 12 unique reads of Orientia tsutsugamushi were detected in the patient's blood and sputum. Quantitative polymerase chain reaction (qPCR) results of blood samples further confirmed our mNGS detection, suggesting that the patient did indeed have scrub typhus. From these results, we determined that mNGS as a diagnostic tool provides a better method of identifying clinical febrile pathogens with atypical characteristics.
\end{abstract}

Keywords: Orientia tsutsugamushi; fever; eschar; metagenomic next-generation sequencing (mNGS); case report

Submitted May 25, 2021. Accepted for publication Jul 07, 2021.

doi: $10.21037 / \mathrm{atm}-21-3015$

View this article at: https://dx.doi.org/10.21037/atm-21-3015

\section{Introduction}

Scrub typhus is an infectious disease caused by a pathogenic bacteria known as Orientia tsutsugamushi (1). This disease is common in Southeast Asia and is reported as a major cause of acute febrile illness in some regions of India and Thailand, especially among children. Cases of scrub typhus typically occur seasonally. In China's Zhejiang Province, its incidence shows a bimodal pattern with two peaks from July to August and from October to November, with an average of 2.2 million people affected by the disease annually (2). While the largest proportion of the disease in Japan happened in November and May. The age distribution of the disease also differs in different regions, such as in China (The largest portion is in 50-60-year-old group, the smallest is in 10-19-year-old group) and in Thailand (The largest portion is in 50-59, 30-39, and 40-49-year-old group) (3). The main symptoms include fever, chills, skin 
rash, and eschar, with the latter being the most typical symptom and often used to diagnose the disease. Patients with severe scrub typhus often experience complications, including jaundice, acute renal failure, pneumonitis, acute respiratory distress syndrome (ARDS), myocarditis, septic shock, meningoencephalitis, pericarditis, and disseminated intravascular coagulation (DIC) (3). Better understanding its epidemiology can improve the ability of clinicians to recognize severe scrub typhus cases and give the patients timely treatment, which decreases the mortality of severe cases. However, if a patient does not display an obvious eschar lesion, it can be difficult to diagnose. This has the potential to compromise the successful treatment of the disease, which requires antibiotics, such as doxycycline or tetracycline (3).

Here, we report a 51-year-old female patient with scrub typhus. In this case, there was no typical eschar on body of the patient, so we did not consider scrub typhus at first. Epidemic hemorrhagic fever of the patient was considered, but negative results for all tests were reported by Zhejiang Provincial Center for Disease Control and Prevention. This led us to use metagenomic next-generation sequencing (mNGS) to detect the pathogen, which was subsequently diagnosed as Orientia tsutsugamushi. It was due to this mNGS result that the patient was then able to be treated for scrub typhus and make a healthy recovery.

We present the following article in accordance with the CARE reporting checklist (available at https://dx.doi. org/10.21037/atm-21-3015).

\section{Case presentation}

The patient was a 51-year-old female farmer who was not known to have suffered from any prior diseases. After experiencing a fever for 1 week, she was admitted to the Emergency Department of Ganzhou Municipal Hospital on December 1, 2020. Prior to being admitted to the hospital, the patient had received treatment for a high fever at another hospital but experienced no improvement in symptoms. The circulation and respiration of the patient were noted to be stable, with a pulse of 107 beats/min, a respiration rate of 21 times per minute, and a body temperature of $39.8{ }^{\circ} \mathrm{C}$. Aside from slight pitting edema of the lower extremities and an anemic appearance, routine examination found no special findings. Scrub typhus was not initially considered due to no rash or eschar appearing on her body. The patient then underwent laboratory tests for platelet count $\left(52 \times 10^{9} / \mathrm{L}\right)$, along with aspartate aminotransferase
(369 IU/L), C-reactive protein $(70 \mathrm{mg} / \mathrm{L})$, and procalcitonin $(2 \mu \mathrm{g} / \mathrm{L})$ levels. We noted that the blood count of the patient had decreased to $2,510 / \mu \mathrm{L}$, but the cause of her infective fever remained unclear. Smears and cultures of blood (December 1-6) and sputum (December 2-6) were performed, but all returned a negative result. The empirical antibiotic treatment of imipenem and cilastatin $(1.0 \mathrm{~g} / 6 \mathrm{~h})$ combined with amikacin $(0.6 \mathrm{~g} /$ day $)$ was administered, but there was no significant decrease in body temperature. Alongside deterioration of the patient's oxygenation index and blood pressure, we also reported a bilateral pulmonary interstitial edema and a small amount of pleural effusion. The blood test results were shown with prothrombin time at 29 seconds, activated partial thromboplastin time at 95.6 seconds, and platelet counts at $6 \times 10^{9} / \mathrm{L}$. The thromboelastography results indicated the presence of platelet hypofunction, so plasma, platelets, and fibrinogen were infused to help restore the patient's blood coagulation function. The patient was transmitted to the ICU on December 3 with her temperature fluctuating around $38{ }^{\circ} \mathrm{C}$. We then proceeded to conduct tests for various diseases, including dengue fever, epidemic hemorrhagic disease, and rheumatic immunity, the results of which were all negative. In addition, the patient's blood, urine, stool, and sputum cultures all provided negative results, and her bone marrow biopsy showed no abnormalities. On December 4, the antibiotic treatment of imipenem and cilastatin was changed to cefoperazone sulbactam $(3.0 \mathrm{~g} / 8 \mathrm{~h})$. We noted that the patient's body temperature dropped but remained higher than normal.

The following day, on December 5, the blood and sputum samples of the patient were sent to undergo PACEseq mNGS analysis (Hugobiotech, Beijing, China). Two days later, the mNGS result revealed that the patient suffered from an Orientia tsutsugamushi infection. The unique sequences of this pathogen were identified in both blood and sputum samples (377 and 12 reads, respectively; Figure 1). Subsequently, we conducted quantitative polymerase chain reaction (qPCR) to detect Orientia tsutsugamushi. The results of these tests further confirmed our mNGS detection (cycle threshold $=32.9$; Figure 1 ). The detailed laboratory tests results and pathogens detection results were shown in Table 1. Following the successful diagnosis of scrub typhus on December 7, the patient's current anti-infective treatment was substituted with minocycline $100 \mathrm{mg} / 12 \mathrm{~h}$. Soon after, the patient's body temperature, platelet, and blood coagulation function returned to normal, and she was discharged from the hospital without suffering any further 


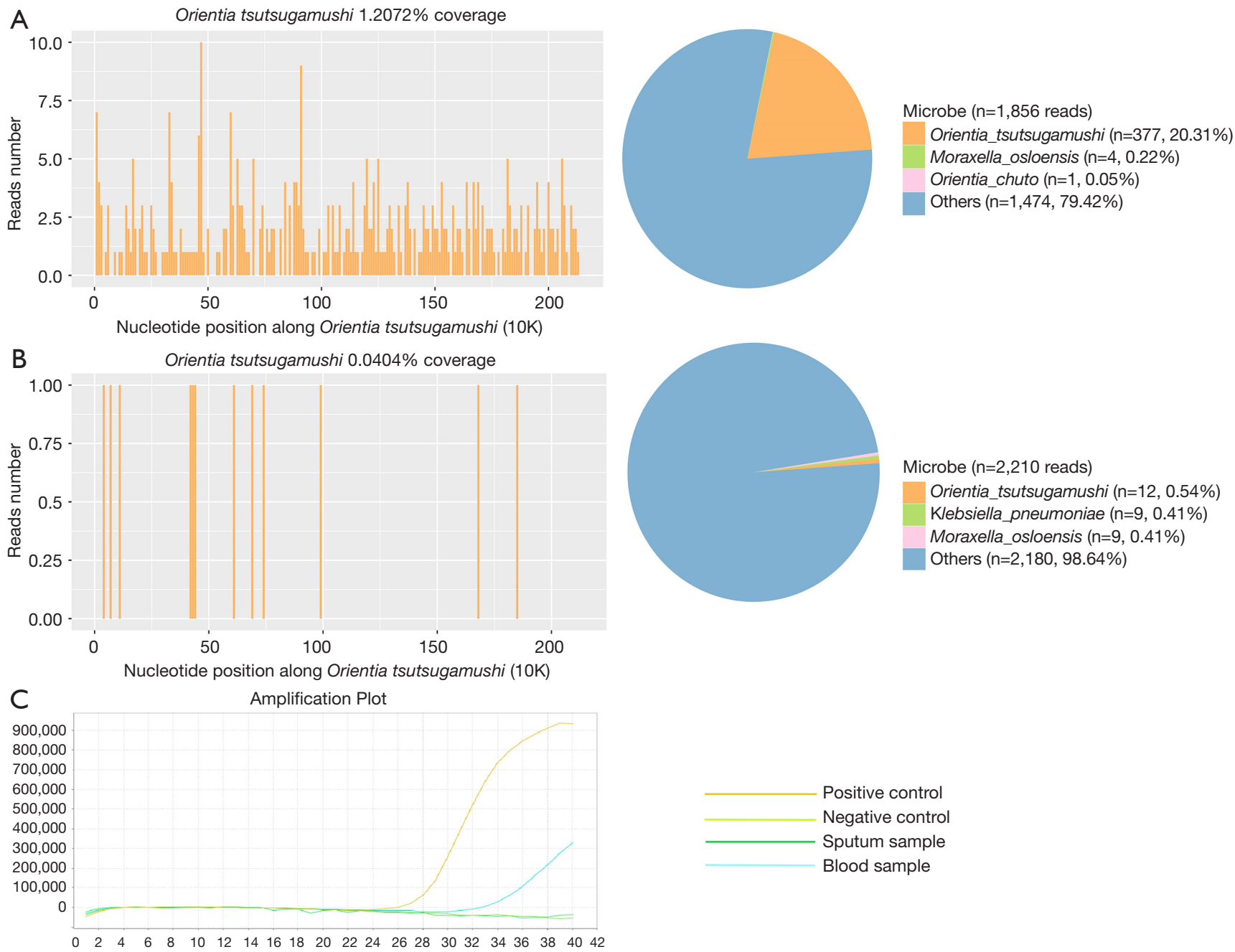

Figure $1 \mathrm{mNGS}$ and qPCR results of the patient. The coverage and proportion of Orientia tsutsugamushi detected by mNGS in blood (A) and sputum (B). The qPCR results of Orientia tsutsugamushi (C). mNGS, metagenomic next-generation sequencing; qPCR, quantitative polymerase chain reaction.

symptoms or complications.

All procedures performed in studies involving human participants were in accordance with the ethical standards of the institutional and/or national research committee(s) and with the Helsinki Declaration (as revised in 2013). Written informed consent was obtained from the patient.

\section{Discussion}

As the patient developed a sudden high fever without any sign of eschar, it was first important to consider her work history. As she was an outdoor worker known to have used pesticides, this led us to first consider liver injury and coagulation disorders. Although there were no direct signs of scrub typhus, the mNGS results correctly identified the disease. Subsequent qPCR analysis further confirmed the diagnosis. Accordingly, we determined that Orientia tsutsugamushi was the pathogen, and minocycline was administered to the patient, whose condition quickly improved.

Scrub typhus often occurs in outdoor workers, especially in farmers (like the patient discussed here). Because of this, it is has been noted as a serious clinical and public health problem (4). Also known as tsutsugamushi disease, scrub typhus usually has an incubation period of 5 to 14 days after exposure (5). In addition to the typical symptom of eschar 
Table 1 Laboratory test results and pathogen detection results by culture and mNGS

\begin{tabular}{|c|c|c|c|c|c|c|c|}
\hline Time & $\begin{array}{c}\text { Leukocyte } \\
\text { counts }\end{array}$ & $\begin{array}{l}\text { Hemoglobin } \\
\text { concentration }\end{array}$ & $\begin{array}{l}\text { Platelet } \\
\text { counts }\end{array}$ & $\begin{array}{l}\text { C-reactive } \\
\text { protein }\end{array}$ & $\begin{array}{c}\text { Creatinine } \\
\text { level }\end{array}$ & BNP & Pathogen detection \\
\hline Dec. 1 (admission) & 8.12 & 54 & 122 & - & 54.1 & - & Blood culture (negative) \\
\hline Dec. 2 & 6.04 & 55 & 194 & - & - & 3,709 & Sputum culture (negative) \\
\hline Dec. 3 & 5.71 & 64 & 158 & 114 & 35 & - & Blood and sputum culture (negative) \\
\hline Dec. 4 & 6.76 & 89 & 249 & 100 & - & 5,663 & Sputum culture (negative) \\
\hline Dec. 7 & 4.78 & 86 & 81 & 12.34 & 61.9 & & mNGS (Orientia tsutsugamushi) \\
\hline Dec. 8 & 2.3 & 73 & 34 & 9.38 & 40.8 & 11,420 & - \\
\hline Dec. 9 & 3.59 & 79 & 37 & - & - & - & - \\
\hline Dec. 10 & 2.97 & 72 & 29 & 4.37 & 68 & 3,505 & - \\
\hline Dec. 14 & 2.86 & 89 & 69 & 42 & 21 & 1,172 & - \\
\hline Dec. 16 (discharge) & - & - & - & - & - & - & - \\
\hline
\end{tabular}

mNGS, metagenomic next-generation sequencing; BNP, brain natriuretic peptide.

at the place of infection, other symptoms include fever, skin rash, and headache (6). It has been noted that $95-100 \%$ of scrub typhus patients display some type of fever (7), while eschar is typically found in up to $97 \%$ of patients from different regions (8). Papule is first formed at the bite site and is followed by the formation of ulcers on the skin (6).

Diagnosis of scrub typhus is usually completed through a serological test and molecular analysis; however, this method faces difficulties when a patient displays no signs of eschar (9). Serologic tests, such as Weil Felix reaction, have a very poor sensitivity, and even need a 4-fold rise in titers in 2 weeks. Indirect immunofluorescence assay (IFA) is the gold standard for diagnosing such diseases (10), but being expensive and difficult to operate, its application is limited. The most common diagnostic method for scrub typhus is qPCR, which performs at a higher sensitivity than IFA (11). qPCR is limited by the fact that it is only used when an Orientia tsutsugamushi infection is suspected beforehand, usually through noticeable eschars.

However, for patients without eschar, scrub typhus is always very hard to be distinguished from other severe fever diseases, such as thrombocytopenia syndrome. mNGS is a new approach and provides an unbiased DNA/RNA detection method through high-throughput and rapid detection. mNGS has been noted to have the capability to detect almost all pathogens regardless of hypothesis or culture and has only recently been used more widely in medical microbiology detection of various diseases, including meningitis, pneumonia, and sepsis (12). It has been proven to be a better technique for detecting pathogens than other, more traditional methods, such as culture, which struggle to detect special or new organisms (12). Despite the limited clinical manifestations of traditional methods in this case, mNGS successfully detected Orientia tsutsugamushi in patient's blood as well as sputum samples.

In addition to scrub typhus, Klebsiella pneumoniae was also detected in the patient's sputum samples with a low confidence value (9 unique reads). However, it was not detected in the blood samples, which means qPCR also failed to identify this pathogen. Due to the scrub typhus diagnosis, Klebsiella pneumoniae infection was eventually not considered in the case.

To conclude, Orientia tsutsugamushi was successfully detected using mNGS in a febrile patient without eschar. Through this diagnostic method, the patient was able to receive immediate treatment and make a full recovery. Given the results of our research, we suggest that mNGS can be a useful method of differentiation for diagnosing fever-related diseases caused by unknown pathogens and especially for patients who do not display typical clinical symptoms. 


\section{Acknowledgments}

Funding: None.

\section{Footnote}

Reporting Checklist: The authors have completed the CARE reporting checklist. Available at https://dx.doi. org/10.21037/atm-21-3015

Conflicts of Interest: All authors have completed the ICMJE uniform disclosure form (available at https://dx.doi. org/10.21037/atm-21-3015). The authors have no conflicts of interest to declare.

Ethical Statement: The authors are accountable for all aspects of the work in ensuring that questions related to the accuracy or integrity of any part of the work are appropriately investigated and resolved. All procedures performed in studies involving human participants were in accordance with the ethical standards of the institutional and/or national research committee(s) and with the Helsinki Declaration (as revised in 2013). Written informed consent was obtained from the patient.

Open Access Statement: This is an Open Access article distributed in accordance with the Creative Commons Attribution-NonCommercial-NoDerivs 4.0 International License (CC BY-NC-ND 4.0), which permits the noncommercial replication and distribution of the article with the strict proviso that no changes or edits are made and the original work is properly cited (including links to both the formal publication through the relevant DOI and the license). See: https://creativecommons.org/licenses/by-nc-nd/4.0/.

\section{References}

1. Kelly DJ, Fuerst PA, Ching WM, et al. Scrub typhus: the geographic distribution of phenotypic and genotypic variants of Orientia tsutsugamushi. Clin Infect Dis 2009;48

Cite this article as: Li J, Chen C, Zou F, Liu L, Wang B, Kang H, Liu B, Wang S, Qin T. Diagnosing scrub typhus without eschar: a case report using metagenomic next-generation sequencing (mNGS). Ann Transl Med 2021;9(14):1190. doi: 10.21037/atm21-3015
Suppl 3:S203-S230.

2. Ren J, Sun J, Wang Z, et al. Re-emergence of scrub typhus in Zhejiang Province, southern China: A 45year population-based surveillance study. Travel Med Infect Dis 2019. [Epub ahead of print]. doi: 10.1016/ j.tmaid.2019.05.013

3. Xu G, Walker DH, Jupiter D, et al. A review of the global epidemiology of scrub typhus. PLoS Negl Trop Dis 2017;11:e006062.

4. Walker DH, Fishbein DB. Epidemiology of rickettsial diseases. Eur J Epidemiol 1991;7:237-45.

5. Paris DH, Shelite TR, Day NP, et al. Unresolved problems related to scrub typhus: a seriously neglected lifethreatening disease. Am J Trop Med Hyg 2013;89:301-7.

6. Mahajan SK. Scrub typhus. J Assoc Physicians India 2005;53:954-8.

7. Tsay RW, Chang FY. Serious complications in scrub typhus. J Microbiol Immunol Infect 1998;31:240-4.

8. Biswal M, Zaman K, Suri V, et al. Use of eschar for the molecular diagnosis and genotypic characterisation of Orientia tsutsugamushi causing scrub typhus. Indian J Med Microbiol 2018;36:422-5.

9. Wilkinson R, Rowland D, Ching WM. Development of an improved rapid lateral flow assay for the detection of Orientia tsutsugamushi-specific IgG/IgM antibodies. Ann N Y Acad Sci 2003;990:386-90.

10. Koraluru M, Bairy I, Varma M, et al. Diagnostic validation of selected serological tests for detecting scrub typhus. Microbiol Immunol 2015;59:371-4.

11. Saisongkorh W, Chenchittikul M, Silpapojakul K. Evaluation of nested PCR for the diagnosis of scrub typhus among patients with acute pyrexia of unknown origin. Trans R Soc Trop Med Hyg 2004;98:360-6.

12. Chen Y, Feng W, Ye K, et al. Application of Metagenomic Next-Generation Sequencing in the Diagnosis of Pulmonary Infectious Pathogens From Bronchoalveolar Lavage Samples. Front Cell Infect Microbiol 2021;11:541092.

(English Language Editors: J. Goetz and J. Gray) 